Deborah Carvalho Malta 1,2

\title{
A mortalidade infantil em Belo Horizonte, Minas Gerais, Brasil, por área de abrangência dos Centros de Saúde (1994-1996)
}

\author{
Analysis of infant mortality in coverage \\ areas of health centers in Belo Horizonte, \\ Minas Gerais, Brazil (1994-1996)
}

Maria Cristina de Mattos Almeida 1

Maria Angélica de Salles Dias 3

Emerson Elias Merhy 2

\footnotetext{
1 Sistema de Informação Secretaria Municipal de Saúde de Belo Horizonte, Av. Afonso Pena 2336, 9o andar, Belo Horizonte, $M G$ 30130-007, Brasil. damata@uol.com.br cristina@horiz.com.br 2 Departamento de Medicina Preventiva e Social, Faculdade de Ciências Médicas, Universidade Estadual de Campinas. C. P. 6111, Campinas, SP 13083-970, Brasil. 3 Núcleo de Epidemiologia, Secretaria Municipal de Saúde de Belo Horizonte, Av. Afonso Pena 2336, 9 o andar, Belo Horizonte, $M G$ 30130-007, Brasil. nucleoep@pbh.gov.br
}

\begin{abstract}
Geographic analysis of health conditions highlights regional and intra-urban inequalities. This study aims to identify intra-urban discrepancies in the city of Belo Horizonte, studying the infant mortality coefficients (IMC) in the various areas of the metropolitan area and evaluating their evolution from 1994 to 1996. The study uses data from 1994 to 1996 regarding birth and death records (from the SINASC and SIM registries). It also uses "thematic maps" related to geographic coverage areas of municipal health centers to demonstrate differences in infant mortality coefficients. The empirical Bayesian method was applied to correct possible random fluctuation in IMC due to small and unstable numbers. A $24.4 \%$ reduction in the overall coefficient was observed in the city from 1994 to 1996. Decreases also occurred in the health center coverage areas. In 1994, eight areas presented an IMC above 50/1,000, while in 1996 no area reached that coefficient. In 1994, 29 areas presented an IMC from 35 to 50/1,000, as compared to only four areas in 1996. In 1996 there were more areas with IMC below 35/1,000 as compared to 1994. In 1996, more homogeneity was observed in IMC distribution and hence a reduction in intra-urban inequalities.
\end{abstract}

Key words Spatial Analysis; Mortality Rate; Infant Mortality

Resumo O trabalho pretende identificar os diferenciais intra-urbanos em Belo Horizonte, Brasil, estudando os coeficientes de mortalidade infantil (CMI) nas áreas do município e avaliando sua evolução no período 1994/1996. A análise das condições de saúde referenciadas territorialmente constitui importante campo de estudo para evidenciar desigualdades regionais e intraurbanas. Para os anos de 1994 e 1996, foram utilizados os dados do SINASC e SIM, além de mapas temáticos com o recorte geográfico da "área de abrangência" dos Centros de Saúde. Aplicouse o método Bayesiano empírico para o cálculo dos CMI dessas áreas, visando corrigir possíveis flutuações aleatórias decorrentes de números pequenos e instáveis. Em todo o período, houve redução do CMI global do município em 24,4\%, assim como do CMI nas áreas de abrangência. Em 1994, oito áreas apresentaram CMI acima de 50/1.000 e, em 1996, nenhuma área foi observada com esse coeficiente; em 1994, vinte e nove áreas mostraram CMI entre 35 a 50/1.000, caindo para quatro áreas em 1996; neste ano, registrou-se, em relação a 1994, maior número de áreas com CMI abaixo de 35/1.000. Em 1996 existiu maior homogeneidade na distribuição dos CMI, reduzindo-se as desigualdades intra-urbanas.

Palavras-chave Análise Espacial; Coeficiente de Mortalidade; Mortalidade Infantil 


\section{Introdução}

Visando examinar os diferenciais intra-urbanos em Belo Horizonte, Brasil, este estudo procurou identificar o Coeficiente de Mortalidade Infantil (CMI) nas diversas áreas desse município. Foram construídos mapas temáticos através do geoprocessamento das informações, o que permitiu a comparação entre as áreas de abrangência da cidade e possibilitou a avaliação do desenvolvimento dessas taxas entre os anos de 1994/1996. Dessa forma, investigou-se a possível existência de sobretaxas em determinadas áreas, contribuindo para a compreensão das causas que levam a desigualdades.

Durante as últimas décadas, mudanças significativas têm ocorrido no que diz respeito à situação da saúde na maioria dos países da América Latina, as quais se traduziram em reduções importantes das taxas de mortalidade específicas nas idades mais jovens, sobretudo em menores de cinco anos. No entanto, por trás da aparente melhoria na realidade sanitária, esconde-se outra, de profundas desigualdades entre países e, no interior de cada país, entre setores sociais. Há fortes evidências de que a desigualdade exerceu impacto negativo nas condições de vida de grandes setores da população durante os anos 80 e início dos 90. Também aumentou o número de pessoas que vivem em condições de pobreza, avaliado em cerca de duzentos milhões na América Latina (Castellanos, 1997).

O tema da desigualdade em saúde mantém-se com a maior atualidade em razão do modelo de desenvolvimento econômico implementado no continente durante a década de 90, que avança acompanhado de uma política social caracterizada por acentuar a exclusão social e a desigualdade, gerando maior contigente de desempregados, menores de rua, sem teto, enfim, de uma massa crescente de excluídos socialmente. Assim, esse tema tornase pauta importante dos movimentos sociais (Barros, 1997), das administrações democráticas populares, ou seja, dos segmentos comprometidos com o avanço das políticas públicas.

Observa-se que a Epidemiologia "descritiva” - descrição e análise de padrões de distribuição de eventos de saúde e, mesmo, de fatores de risco que afetam diferentes segmentos de populações em espaços diversificados - visa à identificação de "diferenças" observáveis em incidências e prevalências de doenças ou taxas de mortalidade. É o achado de "diferenças" que alimenta o desenvolvimento de hipóteses, especulações, análises epidemiológicas, decisões e intervenções (Barros, 1997).
Um campo de estudo que vem se desenvolvendo no sentido de evidenciar as desigualdades conforma-se pela análise das condições de saúde referenciadas territorialmente. Desse modo, a concepção do espaço passa a ser compreendida não só em uma dimensão ecológica, natural ou administrativa, mas na do espaço social e historicamente constituído como expressão de transformações sociais e de formas sociais específicas de ocupação (Assunção et al., 1998; Barros, 1997; Najar \& Marques, 1998; Santos, 1985, 1991).

Diversos autores têm estudado os diferenciais intra-urbanos, buscando identificar as desigualdades, de modo a gerar as possibilidades de sua superação (Akerman, 1997; Barros, 1997; Castellanos, 1997; Granda \& Breil, 1989).

As iniqüidades sociais nos perfis de mortalidade e as condições de vida constituem as principais barreiras ao avanço e à melhoria da situação da saúde. Por conseguinte, as políticas de saúde deveriam considerar não somente os critérios de eficiência, mas também a definição do impacto potencial da redução da mortalidade no que diz respeito à melhoria nas condições de vida das populações marginalizadas (Castellanos, 1997).

Torna-se essencial desenvolver sistemas de análise e de vigilância do efeito advindo das políticas econômicas e sociais na situação da saúde e nas condições de vida dos diferentes setores da população, os quais deveriam vincularse aos níveis decisórios, contribuindo "com os esforços de tornar prioritários os setores e territórios mais empobrecidos, assim como a adequação das intervenções a seus perfis de problemas prioritários" (Castellanos, 1997:149). A lógica concentradora do sistema tenderia a alterar-se e a reverter-se, à medida que os serviços de saúde incorporassem o conceito das desigualdades sociais, assumindo a incumbência das ações relativas a populações social e territorialmente definidas (Barros, 1997).

\section{Análise dos dados espaciais em saúde e monitoramento das desigualdades}

Em 1854, John Snow descrevia que a mortalidade pela cólera em Londres era mais elevada nas residências servidas por uma das companhias que abastecia a população de água, captando-a diretamente do Rio Tâmisa, abaixo do ponto onde eram lançados os esgotos da cidade. Dessa maneira, quase meio século antes das descobertas da microbiologia, a incorporação da categoria "espaço" na análise da distribuição da doença permitiu a hipótese de asso- 
ciação causal (Rosen, 1994). Modernamente, foram introduzidos outros conhecimentos, como, por exemplo, a geografia, desenvolvendo-se técnicas e modelos estatísticos de análise da distribuição espacial das doenças. Assim, o geoprocessamento vem surgindo como campo promissor, auxiliando a Epidemiologia e a Saúde Pública no que concerne à compreensão dos fenômenos relacionados à dinâmica da distribuição das doenças (Najar \& Marques, 1998).

Segundo Assunção et al. (1998), os mapas temáticos configuram poderosos instrumentos na análise espacial do risco de determinada doença, podendo-se listar três objetivos principais: o primeiro, consta da descrição e simples visualização da distribuição espacial do evento na região de interesse; o segundo, exploratório, consiste em sugerir os determinantes locais do evento e fatores etiológicos desconhecidos que possam ser formulados em termos de hipóteses a serem investigadas posteriormente; o terceiro objetivo seria o de apontar associações entre o evento e seus determinantes.

Utilizando os mapas temáticos no estudo da distribuição da mortalidade infantil em Municípios de Minas Gerais, Assunção et al. (1998) concluem pela forte correlação espacial entre as taxas na situação em que municípios vizinhos tendem a ter taxas mais similares, permitindo, por exemplo, identificar facilmente bolsões com taxas elevadas e outros, com taxas baixas, o que mostra a desigualdade existente entre os diversos municípios mineiros. Entretanto, o mapa também apresenta seus problemas, como, por exemplo, a presença de algumas localidades com valores distintos e extremos em relação aos de seus vizinhos. Esses valores discrepantes podem ser causados por flutuação aleatória sem associação com o risco subjacente. Quando o número de nascidos vivos é pequeno, a taxa pode sofrer variações importantes em virtude da pequena mudança no número de óbitos. Esse grau de variabilidade está ligado ao tamanho das unidades geográficas de análise, o que acarretará estimativas de risco instáveis em pequenas populações, impedindo assim a comparação e utilização desse poderoso instrumento - o mapa temático no estudo de pequenas áreas.

Uma alternativa a essa dificuldade seria o emprego de mapas subdivididos em áreas de análises maiores por meio de agregação das áreas menores. Contudo, isso acarretaria perda de informação, já que um dos principais objetivos ao serem elaborados os mapas temáticos seria a identificação de desigualdades em áreas geográficas menos agregadas, detectando áreas de risco para orientar intervenções de saúde pública. Para superar essa dificuldade, Assunção et al. (1998) utilizam o método Bayesiano. Este método, ao estimar o risco de uma pequena área, tem, como idéia central, o uso de informações das outra áreas que compõem a região de estudo, para diminuir o efeito das flutuações aleatórias não associadas ao risco. Os valores discrepantes resultantes podem ser estudados sem o temor de haver ocorrido flutuação aleatória causada por populações pequenas, resultando em mapas mais confiáveis e fáceis de interpretar. O método, entretanto, não afeta erros sistemáticos, como aqueles causados por sub-registros, e isso envolve que a sua interpretação requer conhecimento a respeito dos dados e das áreas estudadas (Assunção et al., 1998).

\section{Material e métodos}

Em 1992, foi implantado o Sistema de Informação sobre Nascidos Vivos (SINASC) na Secretaria Municipal de Saúde (SMS). Como conseqüência, todos os nascimentos ocorridos nas Maternidades do município passaram a ser comunicados à SMS através das Declarações de Nascidos (DN). Esse procedimento permitiu a montagem do banco de dados relativo aos nascidos vivos, tornando possível a utilização de denominadores confiáveis e a detecção de recém-nato $(\mathrm{RN})$ com maior risco de adoecer e morrer.

Belo Horizonte é dividido em nove Distritos Sanitários (DS), que se subdividem em áreas de abrangência dos Centros de Saúde. A definição dessas áreas foi realizada a partir do processo de territorialização havido em 1992, atentando-se para o acesso da população aos serviços, suas barreiras geográficas e as malhas viárias. Em seu desenho, essas áreas levaram em consideração os limites dos setores censitários definidos pela Fundação Instituto Brasileiro de Geografia e Estatística (IBGE) e digitalizados pela Empresa de Processamento de Dados do município (Prodabel). Em cada área de abrangência, foram identificadas áreas de risco de acordo com critérios como: tipo de moradias, condições de infra-estrutura urbana caracterizadas pela presença ou não de água tratada, esgotamento sanitário, pavimentação, iluminação, riscos geológicos e outros riscos sócioeconômicos já identificados por outros setores da administração municipal. Estas informações foram complementadas pelas equipes técnicas dos Centros de Saúde, que utilizaram pesquisas do tipo "estimativa rápida" para consolidar as informações acerca de determinado 
território com base em fontes tais como diversas formas de registros, entrevistas com informantes chaves da comunidade e observação direta (Mendes, 1994; Unglert, 1994; Veloso, 1995). As áreas foram digitalizadas, passando a integrar a base geográfica do município. Através de um módulo complementar aos sistemas nacionais para registro de nascimentos (SINASC), de óbitos (Sistema de Informação sobre Mortalidade - SIM) e da base geográfica do município, tornou-se possível o geo-referenciamento de nascidos, casos sob vigilância, óbitos, residentes em áreas de risco, dentre outros. A variável “área de abrangência” de residência foi incluída no banco de dados a partir de 1995.

A SMS, por meio do Núcleo de Epidemiologia (Nepi), estruturou o banco de dados do SIM nos anos de 1993 e 1994, iniciando pela digitação de cópias xerográficas das declarações de óbito obtidas na Fundação João Pinheiro (FJP), órgão estadual responsável pelo sistema de informação de mortalidade nessa época. Após esse período, as declarações passaram a ser entregues diretamente na SMS, e o banco de dados de mortalidade veio a ser sistematicamente trabalhado, melhorando-se a qualidade dos endereços; desse modo, os coeficientes atuais espelham a realidade de forma mais precisa.

A cobertura do SINASC para Belo Horizonte após 1993 tem sido próxima dos 100\%, já que no SINASC, em 1996, 99,8\% dos partos foram hospitalares, tendo sido emitida a DN em todos os estabelecimentos, ao passo que, nos demais $(0,2 \%)$, os próprios cartórios a providenciam no momento do registro de nascimento (Rodrigues et al., 1997). No caso do SIM, também é obrigatória a emissão da Declaração de Óbito (DO), sem a qual não é permitido o sepultamento. Não se tendo conhecimento de cemitérios clandestinos em Belo Horizonte, estima-se a cobertura do SIM em cerca de $100 \%$.

Para o estudo atual, foram utilizados os dados do SINASC e do SIM dos anos de 1994 e de 1996. A delimitação das áreas de abrangência refere-se a definições estabelecidas no ano de 1995. Foram analisadas 118 áreas em 1994, ao passo que, em 1996, foram incluídas três novas unidades, totalizando, portanto, 121 áreas estudadas.

Como no ano de 1994 não existia o registro da área de abrangência, os endereços de RN e os óbitos foram geo-referenciados com base em endereços e logradouros da Prodabel, o que possibilitava a posterior identificação da área de abrangência correspondente. Em 1.411 registros do SIM, 136 não foram geo-referenciados $(9,6 \%)$. No banco de dados do SINASC, mesmo após o geo-referenciamento, permaneceu significativo número de registros com área de abrangência ignorada, concentrados no Distrito Noroeste. Assim, procedeu-se à distribuição proporcional dos 1.537 nascidos entre as áreas de abrangência do referido distrito, levando em consideração a contagem populacional dos menores de cinco anos de 1996 (IBGE, 1996). Em 1994, os registros sem área de abrangência determinada no SINASC foram de 5,9\% (2.443/ 41.400).

A partir de 1995, a variável “área de abrangência” passou a ser informada pelos técnicos dos Distritos Sanitários mediante o conhecimento das ruas, de bairros e de mapas dessas regiões.

Em 1996, tanto para o SIM quanto para o SINASC, empregou-se o geo-referenciamento dos registros por endereço apenas para complementar aqueles nos quais a "área de abrangência” de residência não estava informada, permanecendo $0,04 \%$ (15/41.489) como ignoradas no SINASC e $0,09 \%$ ( $1 / 1.055)$, no SIM.

Utilizou-se o software Excel para o cálculo dos CMI das "áreas de abrangência”. Em 1994, nasceram 41.406 crianças em Belo Horizonte, das quais 1.411 foram a óbito com menos de um ano de vida. Em 1995, nasceram 42.153 crianças; destas, 1.205 foram a óbito com menos de um ano de vida; por fim, em 1996, nasceram 41.487 crianças e, dentre estas, 1.055 foram a óbito com menos de um ano de vida.

No software MapInfo (MapInfo Corporation, 1994) foram feitos mapas temáticos, comparando-se os coeficientes de mortalidade infantil por DS e por área de abrangência. Os CMI por DS, em 1994, foram agregados em quatro categorias (28,6 a 30,7/1.000; 30,7 a 32,3/1.000; 32,3 a 33,4/1.000; 33,4 a 34,7/1.000) com faixas pouco amplas. Aqueles referentes às áreas de abrangência foram agrupados em cinco categorias: mortalidade menor e igual a 10/1.000; mortalidade entre 10 e 20/1.000; mortalidade entre 20 e 35/1.000; mortalidade entre 35 e $50 / 1.000$; e, por fim, mortalidade maior que $50 / 1.000$.

Para a estimativa dos coeficientes de mortalidade infantil das áreas de abrangência dos Centros de Saúde, empregou-se a abordagem Bayesiana empírica global, com o objetivo de corrigir as possíveis distorções que ocorrem quando se trabalha com números pequenos e instáveis. Esse método consiste em ajuste dos CMI por área em direção ao coeficiente do município através de um fator de correção que contempla a variabilidade dessas taxas e o número de nascidos em cada área, de acordo com a seguinte fórmula (Bailey \& Gatrell, 1995): 
$\mathrm{CMIe}_{\mathrm{i}}=\mathrm{w}_{\mathrm{i}} * \mathrm{r}_{\mathrm{i}+}\left(1-\mathrm{w}_{\mathrm{i}}\right) * \mathrm{R}$

Onde CMIe $_{\mathrm{i}}$ é o coeficiente de mortalidade infantil estimado para a área de abrangência ${ }_{i}$;

$\left(r_{i}\right)$ é o coeficientes de mortalidade infantil usual da área;

(R) é o coeficientes de mortalidade infantil do município;

$\left(\mathrm{w}_{\mathrm{i}}\right)$ é o peso, ou fator de correção da área ${ }_{\mathrm{i}}$, que é calculado através de:

$\mathrm{w}_{\mathrm{i}}=\mathrm{v} /\left(\mathrm{v}+\mathrm{R} / \mathrm{n}_{\mathrm{i}}\right)$

onde:

$\left(\mathrm{n}_{\mathrm{i}}\right)$ é o número de nascidos na área;

(v) expressa a variabilidade dos coeficientes, sendo calculado segundo:

$$
\mathrm{V}=\frac{\sum\left[\left(\mathrm{n}_{\mathrm{i}}\left(\mathrm{R}-\mathrm{r}_{\mathrm{i}}\right)^{2}\right]\right.}{\mathrm{N}}-\frac{\sum \mathrm{r}_{\mathrm{i}}}{(\mathrm{N} / \mathrm{A})}
$$

onde:

(N) é o total de nascidos do município;

(A) é o número de áreas de abrangência.

Esses cálculos foram feitos em planilha do Excel, produzindo-se um coeficiente de mortalidade infantil ponderada para cada área de abrangência, com as quais foram feitas as comparações entre 1994 e 1996.

\section{Resultados}

Em Belo Horizonte, no período entre 1979 a 1996, houve diminuição do CMI, que passou de 64,02, em 1979, para 25,8 óbitos por 1.000 nascidos vivos, em 1996 (Tabela 1), seguindo a tendência da maioria das capitais do Brasil e da América Latina (Simões \& Monteiro, 1995). Semelhante queda deveu-se à redução dos óbitos infantis tardios por diarréias, pneumonias e desnutrição. No período do estudo, ocorreu decréscimo do CMI, que passou de 34,1/1.000, em 1994, para 25,8/1.000, em 1996, representando redução de $24,4 \%$ no coeficiente.

Ao analisar as áreas dos nove DS que compõem o município e que correspondem, cada um deles, a um contingente de cerca de 250 a 300 mil habitantes, não se notam grandes diferenciais na distribuição da mortalidade entre essas áreas. Da mesma forma que o observado para o município, nessas áreas também há redução do CMI.

A Figura 1 mostra que os coeficientes entre os distritos distribuíam-se, em 1994, entre 28,6/ 1.000 - menor CMI no DS Noroeste - e 34,7/ 1.000 - maior CMI no DS Norte. Embora exista diferença entre os distritos, as realidades geográficas de análise ainda são muito agregadas,

\section{Tabela 1}

\begin{tabular}{ll} 
Série histórica dos coeficientes de mortalidade \\
infantil por 1.000 nascidos vivos. Belo Horizonte, \\
Minas Gerais, Brasil - 1979 a 1996. \\
\hline \multicolumn{2}{c}{ Coeficiente de Mortalidade Infantil } \\
\hline 1979 & 64,0 \\
1980 & 57,4 \\
1981 & 54,1 \\
1982 & 54,8 \\
1983 & 46,5 \\
1984 & 44,8 \\
1985 & 42,5 \\
1986 & 38,7 \\
1987 & 41,7 \\
1988 & 43,9 \\
1989 & 36,6 \\
1990 & 36,5 \\
1991 & 36,7 \\
1992 & 39,2 \\
1993 & 34,4 \\
1994 & 34,1 \\
1995 & 28,5 \\
1996 & 25,8 \\
\hline
\end{tabular}

Fontes: Secretaria de Planejamento (SEPLAN) - 1979 a 1992; Sistema de Mortalidade/Ministério da Saúde - 1987 a 1992; Núcleo de Epidemiologia/Secretaria Municipal de Saúde de Belo Horizonte - 1993-1995.

dificultando estudos e intervenções. Em 1996 ocorreu redução do CMI nos distritos, mas os coeficientes distritais permaneceram próximas do coeficiente global do município, variando entre 20 e $30 / 1.000$.

Visando avaliar as desigualdades intra-urbanas por intermédio do enfoque de áreas menores e mais homogêneas, estudou-se a evolução da mortalidade infantil no período, localizando com maior detalhamento as mudanças entre regiões menores (as áreas de abrangência). Essas áreas constituem territórios mais homogêneos do ponto de vista do comportamento dos indicadores de morbi-mortalidade. Calculando-se os CMI de forma mais desagregada, torna-se possível avaliar as diferenças ocorridas em áreas menores e propor as intervenções necessárias.

Com a aplicação do método Bayesiano, nota-se tendência à aproximação dos coeficientes de mortalidade infantil de áreas ao CMI do município, que se expressa de forma mais evidente naquelas áreas com CMI extremos (superior ou inferior). A Figura 2 compara o CMI de 1994 usual com o CMI de 1994 estimado pelo método Bayesiano empírico. Os valores do 
Figura 1

Coeficiente de mortalidade infantil, por Distrito Sanitário. Belo Horizonte, 1994

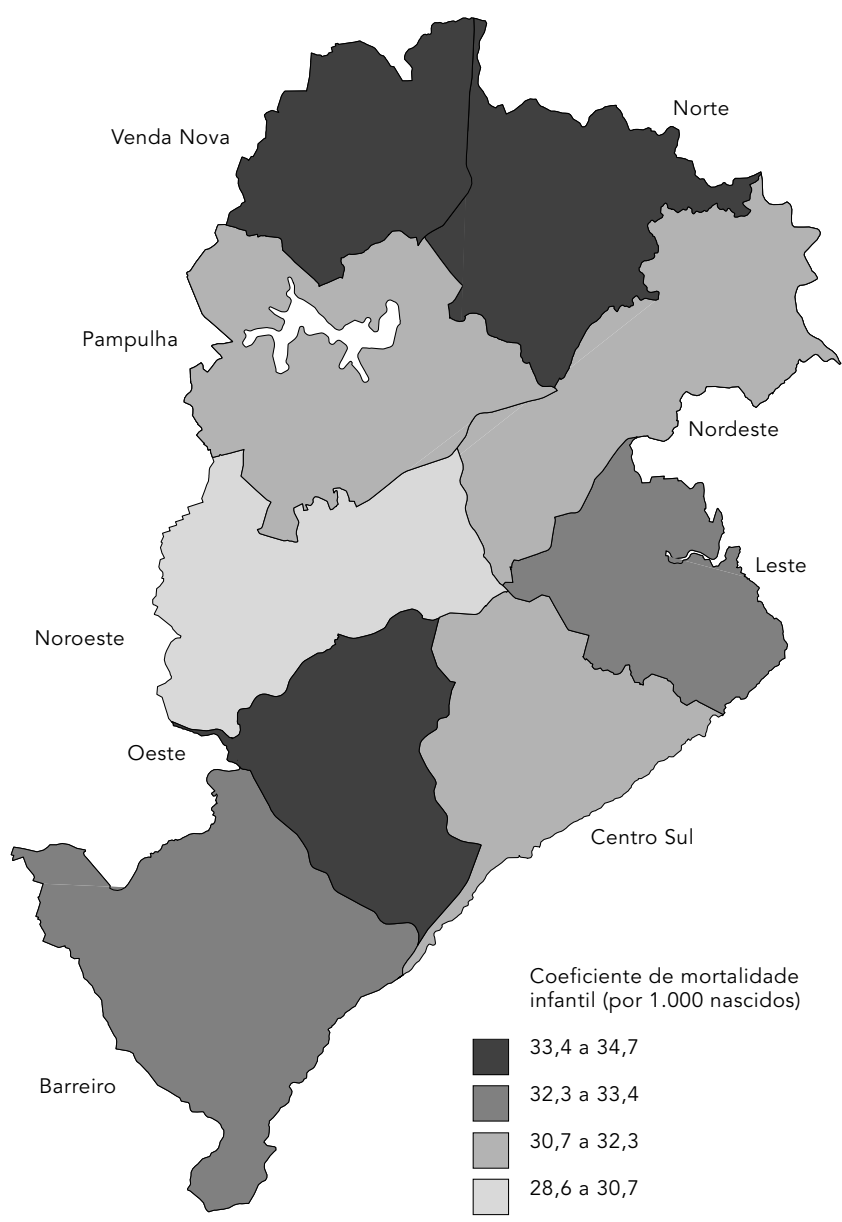

Fonte: Sistema de Informação sobre Mortalidade, 1994; Sistema de Informação sobre Nascidos Vivos, 1994; Núcleo de Epidemiologia/Departamento de Planejamento e Regulação, Secretaria Municipal de Saúde de Belo Horizonte. Dados geográficos: Empresa de Processamento de Dados do Município. acima desse patamar dá-se queda acentuada no CMI no confronto do ano de 1994 com o de 1996. A figura mostra também propensão à homogeneização do CMI no município sem discrepância entre as diversas áreas.

Semelhantes informações são melhor percebidas através dos mapas temáticos. A Figura 4 compara os coeficientes de mortalidade infantil de 1994 e 1996 estimados por área de abrangência. Em 1994, 8 áreas apresentavam coeficiente maior que 50/1.000 nascidos vivos; 29 áreas, entre 35 a 50/100; 66 áreas, entre 20 a 35/1.000; em 14 áreas, os coeficientes estavam entre 10 a 20/100 e uma área, com coeficiente abaixo de 10/1.000. O cálculo do coeficiente de mortalidade infantil em grandes áreas (Distritos Sanitários) ocultava esses diferenciais.

Ao comparar o CMI com 1996, vê-se que quatro áreas apresentaram coeficientes entre 35 a 50/100; 96 áreas, entre 20 a 35/1.000; por fim, 19 áreas, entre 10 a 20/1.000. Não foi observada nenhuma área acima de 50/1.000. Nota-se mudança do perfil de mortalidade em Belo Horizonte com melhoria nas condições de saúde da população infantil, situação evidenciada não só pela redução global do coeficiente do município, mas também pela redução do indicador de mortalidade infantil em áreas menores, registrando-se maior homogeneidade na distribuição dos CMI no município, o que comprova, por sua vez, a redução de desigualdades intra-urbanas.

\section{Discussão}

Os indicadores de mortalidade, embora sejam os mais usados em saúde pública, ainda sofrem distorções relacionadas à qualidade dos serviços de registros de dados vitais e ao subregistro no interior do Brasil. Nesse sentido, a estruturação de sistemas nacionais de informação como o SIM e SINASC contribuiu para a melhora da qualidade e da confiabilidade dos registros, aliada ao fato de que, nas grandes cidades, o sub-registro de óbitos tem sido mais controlado (Carvalho, 1997). Em Belo Horizonte, com a implantação e análise dos bancos do SINASC e SIM no município a partir de 1993, passou a existir maior confiabilidade nos dados, além do aperfeiçoamento da cobertura (Rodrigues et al., 1997). Apesar dos avanços, houve problemas nos dados em 1994, exemplificados pelo seguinte percentual de registros com procedência ignorada: 5,9\% para o SINASC e 9,6\% para o SIM, o que pode resultar em erros sistemáticos. Em 1996, ocorreu diminuição importante do número de registros ignorados. 


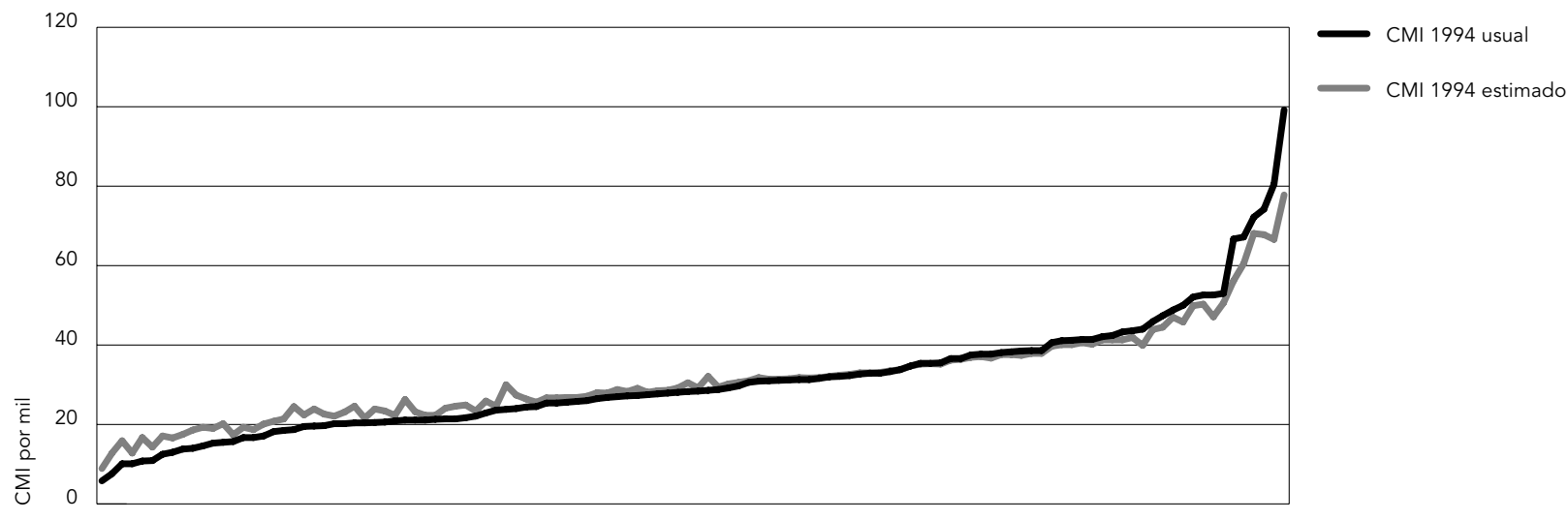

áreas de abrangência

Figura 3

Coeficiente de mortalidade infantil (CMI). Belo Horizonte, 1994 e 1996.

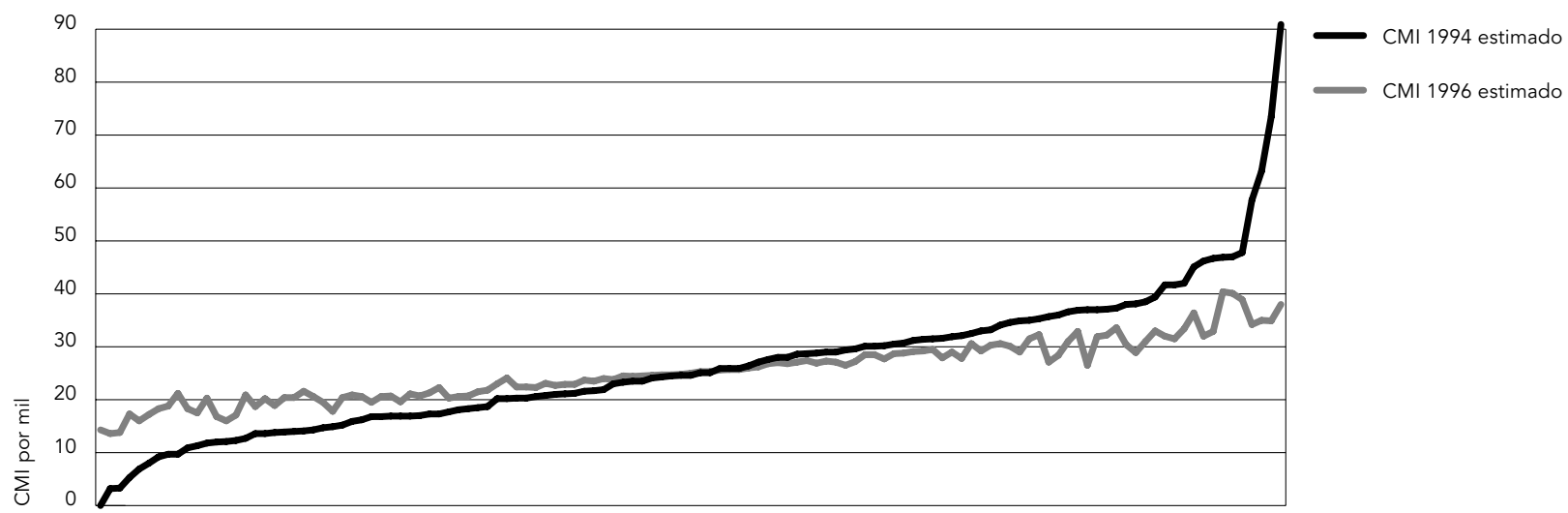

áreas de abrangência

No estudo de Barreto et al. (1997) são descritas as atuais tendências declinantes da mortalidade infantil em várias sociedades, dissociadas de mudanças dos indicadores sociais e econômicos. Entretanto, a mortalidade infantil permanece como razoável indicador de desigualdades regionais, expressando os desníveis de saúde, as iniqüidades e os diferenciais intraurbanos (Almeida Filho \& Rouquayrol, 1992; Barros, 1997; Monteiro \& Nazário, 1995).

No Brasil, vários estudos têm demonstrado a existência de diferenciais de mortalidade in- fantil tanto entre estratos sociais (Simões, 1992; Victora et al., 1988), como entre regiões do país (Simões \& Monteiro, 1995) e áreas da mesma cidade (Monteiro, 1988; Monteiro et al., 1980; Paim et al., 1987). O presente estudo também procura identificar esses diferenciais dentro do mesmo município através do estudo dos CMI das diversas áreas de abrangência.

Existem diversos recortes espaciais possíveis do município, tais como o Índice de Qualidade de Vida Urbano (IQVU), que estuda as desigualdades regionais nas 80 Unidades de Pla- 


\section{Figura $4 a$}

Coeficiente de mortalidade infantil, por área de abrangência dos centros de saúde Belo Horizonte, 1994

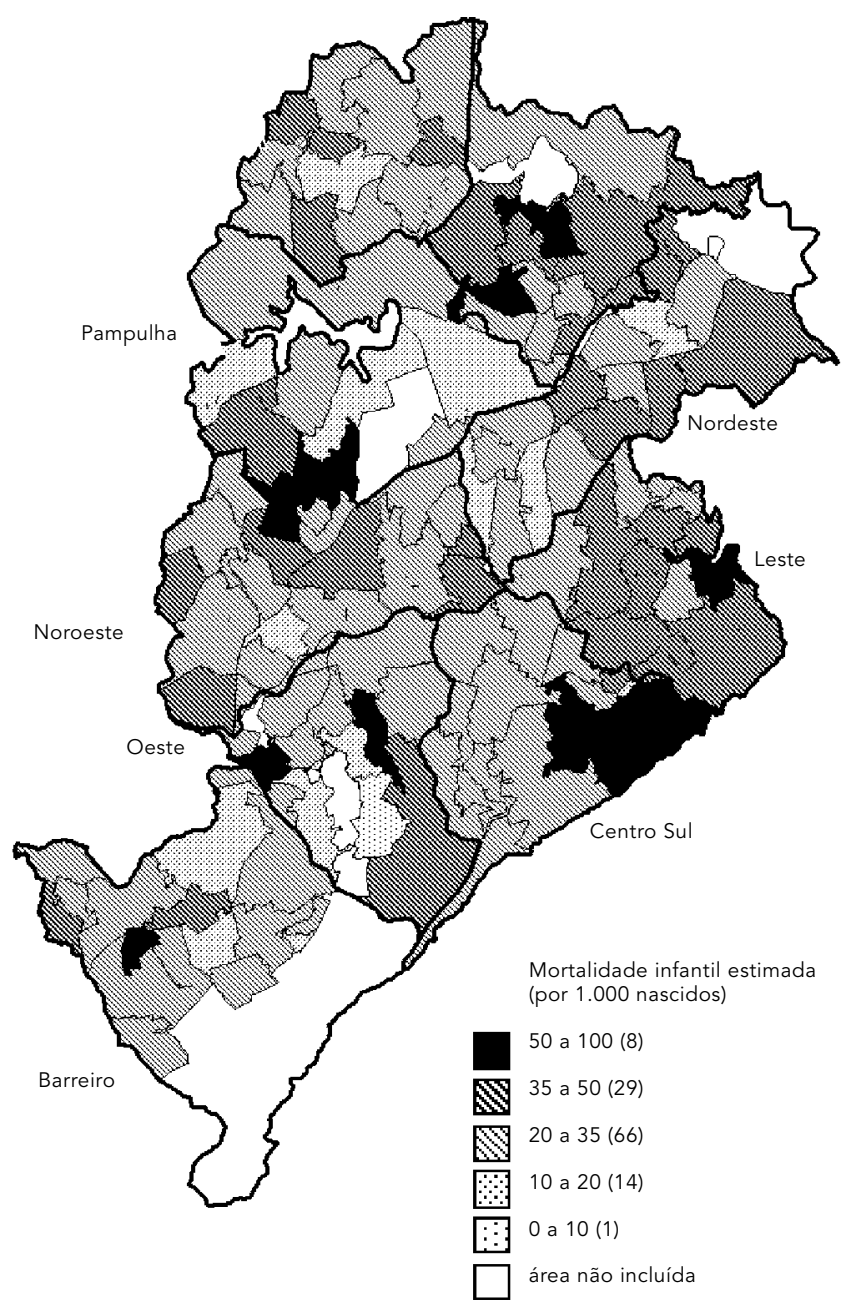

Fonte: Sistema de Informação sobre Mortalidade/Ministério da Saúde, 1994; Núcleo de Epidemiologia/Departamento de Planejamento e Regulação, Secretaria Municipal de Saúde de Belo Horizonte.

Dados geográficos: Empresa de Processamento de Dados do Município. nejamento (UP), e o Índice de Condição de Vida (ICV) da Fundação João Pinheiro, que aborda as diferenças regionais. Todas essas unidades geográficas foram construídas a partir do setor censitário (FJP, 1996; SMPL, 1996). O atual trabalho utilizou mapas temáticos com o recorte geográfico das "áreas de abrangência” no estudo dos diferenciais intra-urbanos da mortalidade infantil, visando facilitar o planejamento local pela delimitação de território do Centro de Saúde, ou seja, do espaço geográfico onde se exerce o papel de promoção à saúde, vigiando-se os óbitos e outros eventos considerados evitáveis.

Ao aplicar o método Bayesiano empírico global, os mapas tornaram-se mais informativos, estimando o risco das áreas de maneira mais precisa, permitindo análise com maior segurança (Assunção et al., 1998). Nesta pesquisa recorreu-se ao CMI do município para a estimativa do CMI das áreas. Entretanto, avaliou-se posteriormente ser necessário utilizar a estimativa Bayesiana por vizinhança, ou seja, realizar as correções por áreas próximas e estabelecer a comparação com o reajuste empregado no trabalho.

O estudo mostrou ainda a importância do trabalho com dados de forma desagregada por permitir a localização espacial em pequenas unidades, o que possibilita evidenciar as desigualdades acentuadas entre as diversas regiões da cidade (Akerman, 1997; Barros, 1997; Monteiro, 1988; Monteiro et al., 1980; Paim et al., 1987), comprovando-se o risco diferenciado das populações residentes em vilas e favelas (áreas de risco) na mortalidade infantil em Belo Horizonte (Malta et al., 1998).

Os resultados exibem redução dos coeficientes de mortalidade infantil em todos os DS e na maioria das áreas de abrangência das unidades de saúde do Município de Belo Horizonte. A contribuição maior do estudo atual foi a de identificar os diferenciais intra-urbanos no município, evidenciando as diferenças entre os coeficientes de mortalidade infantil em suas diversas áreas e mostrando a evolução e a existência de sobretaxas em determinadas áreas, o que contribui para a percepção de que a cidade não é um todo homogêneo, mas que apresenta desigualdades, e coloca a necessidade de um planejamento em saúde que contemple tais dimensões.

Houve redução significativa do CMI em determinadas áreas, muitas das quais consideradas áreas de risco, o que possibilitou à cidade tornar-se menos heterogênea na distribuição da mortalidade infantil. Por meio do mapeamento dessas desigualdades, estratégias diferenciadas de intervenção podem ser traçadas de modo a haver aprendizagem com base nos erros e dificuldades encontradas na execução das políticas e programas, além de avaliar se as ações em curso têm conseguido reduzir as desigualdades, retornando as informações para os atores diretamente envolvidos - trabalhadores e usuários -, permitindo que os resultados iluminem a intervenção para mudar rumos e rever estratégias. Em uma forma mais renovada de entender a avaliação das políticas sociais, esta é entendida, enquanto poderoso instru- 
mento para melhorar o desempenho dos programas sociais, como instância de aprendizagem que permite reelaborar e melhorar esses programas (Arretche, 1998; Sulbrandt, 1994).

A adoção do enfoque de risco pelos serviços de saúde em Belo Horizonte tornou possível a análise dos problemas dessa área de atuação, a definição de prioridades e o direcionamento de ações aos grupos sociais mais dramaticamente excluídos do atendimento. No caso do combate à mortalidade infantil, o Projeto de Vigilância à Mortalidade Infantil - implantado na rede pública municipal em 1994 passou a identificar como grupos sob vigilância e em acompanhamento pelos Centros de Saúde aqueles recém-nascidos residentes em áreas de risco e que somavam um dos seguintes critérios: baixo peso (menor que $2.500 \mathrm{~g}$ ), filho de mãe analfabeta ou adolescente (Malta et al., 1998). Estas variáveis eram identificadas através do SINASC e foram selecionadas por serem fatores já relatados na literatura como favorecedores de maior risco de óbito (Victora et al., 1988).

A redução do CMI deu-se de forma expressiva nas regiões de vilas e favelas, o que pode ser atribuído a iniciativas e à vigilância no tocante à mortalidade infantil, bem como à melhoria na atuação dos serviços de saúde, na urbanização e saneamento, dentre outras. $\mathrm{O}$ atual estudo não consegue isolar o efeito das diferentes variáveis e o seu comportamento na redução da mortalidade infantil, mas permite verificar a queda da mortalidade infantil e das desigualdades no município, tornando-o menos desigual em curto espaço de tempo. Mesmo diante das limitações que apresenta, torna-se importante este tipo de análise avaliativa, que possibilita tanto a tradução dessas mudanças para as equipes dos Centros de Saúde quanto a ampliação da responsabilidade sanitária na condução das ações de saúde em âmbito local.

\section{Agradecimentos}

Nossos agradecimentos ao Núcleo de Epidemiologia da Secretaria Municipal de Saúde pelo apoio durante as discussões do trabalho.

\section{Figura 4b}

Coeficiente de mortalidade infantil, por área de abrangência dos centros de saúde. Belo Horizonte, 1996.

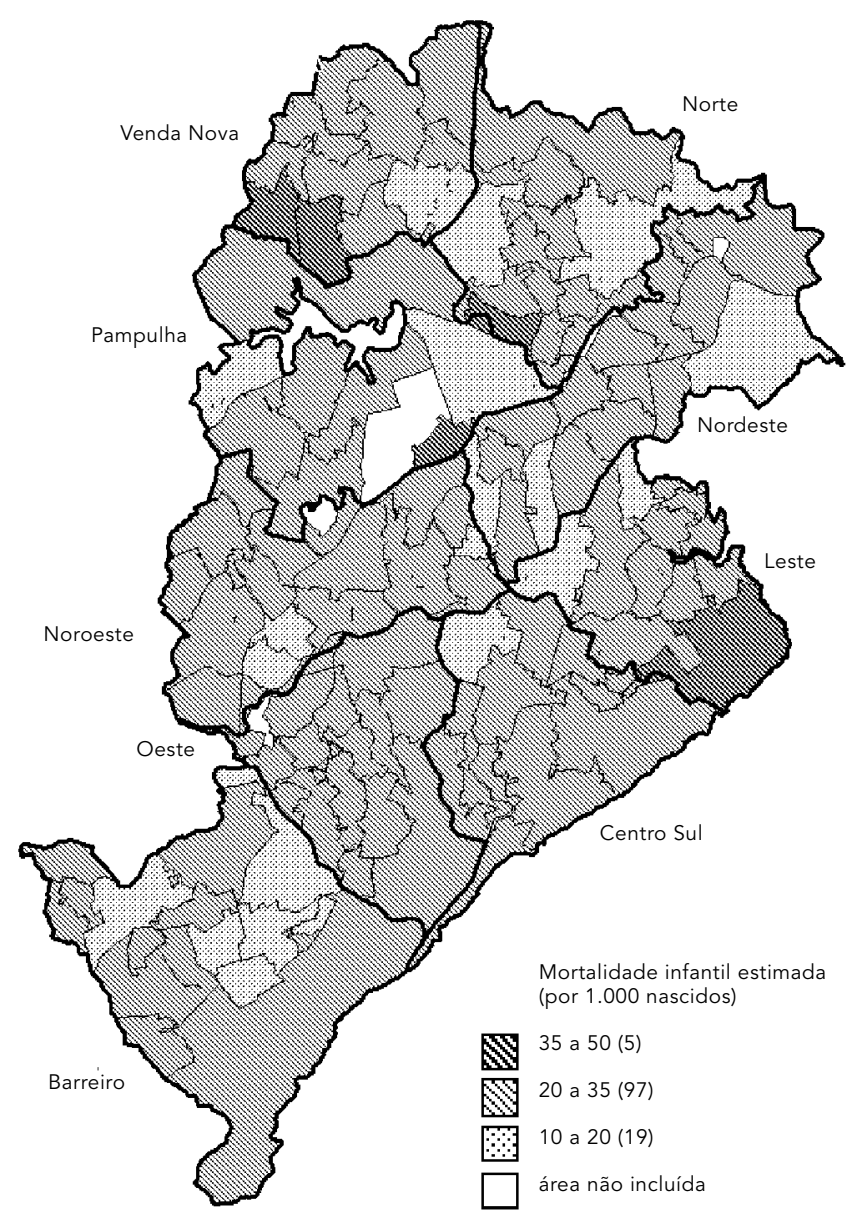

Fonte: Sistema de Informação sobre Mortalidade, 1996; Sistema de Informação sobre Nascidos Vivos, 1996 - Núcleo de Epidemiologia/Departamento de Planejamento e Regulação, Secretaria Municipal de Saúde de Belo Horizonte. Dados geográficos: Empresa de Processamento de Dados do Município.

\section{Referências}

AKERMAN, M., 1997. Diferenciais intra-urbanos em São Paulo: Estudo de caso de macrolocalização de problemas de saúde. In: Eqüidade e Saúde, Contribuições da Epidemiologia (R. B. Barata, M. L. Barreto \& N. Almeida Filho, org.), pp. 177-186, Série Epidemiologia 1, Rio de Janeiro: Editora Fiocruz/ABRASCO.

ALMEIDA FILHO, N. \& ROUQUYROL, M. Z., 1992. Introdução à Epidemiologia Moderna. Belo Horizonte: Editora Coopmed, APCE/Rio de Janeiro: ABRASCO.

ARRETCHE, M. T. S., 1998. Tendências no estudo sobre avaliação. In: Avaliação de Políticas Sociais: Uma Questão em Debate (E. M. Rico, org.), pp. 29-39, São Paulo: Editora Cortez/Instituto de Estudos Especiais. 
ASSUNÇ̃̃O, R. M.; BARRETO, S. M.; GUERRA, H. L. \& SAKURAI, E., 1998. Mapas de taxas epidemiológicas: Uma abordagem Bayesiana. Cadernos de Saúde Pública, 14:713-723.

BAILEY, T. C. \& GATRELL, A. C., 1995. Interactive Spatial Data Analysis. Essex: Longman Scientific \& Technical.

BARRETO, M. L.; CARMO, E. H. \& SANTOS, C. A. S. T. S., 1997. Saúde e população brasileira: Mudanças, superposição de padrões e desigualdades. In: Saúde e Democracia, a Luta do CEBES (S. Fleury, org.), pp. 45-60, São Paulo: Editora Lemos.

BARROS, M. B. A., 1997. Epidemiologia e superação das iniqüidades em saúde. In: Eqüidade e Saúde, Contribuições da Epidemiologia (R. B. Barata, M. L. Barreto \& N. Almeida Filho, org.), pp. 161-176, Série Epidemiologia 1, Rio de Janeiro: Editora Fiocruz/ABRASCO.

CARVALHO, D. M., 1997. Grandes sistemas nacionais de informação em saúde: Revisão e discussão da situação atual. Informe Epidemiológico do SUS, 4:7-46.

CASTELLANOS, P. D., 1997. Epidemiologia, saúde pública, situação de saúde e condições de vida. Considerações conceituais. In: Eqüidade e Saúde, Contribuições da Epidemiologia (R. B. Barata, M. L. Barreto \& N. Almeida Filho, org.), pp. 137-160, Série Epidemiologia 1, Rio de Janeiro: Editora Fiocruz/ABRASCO.

FJP (Fundação João Pinheiro), 1996. Índice de Condição de Vida nos Município de Minas Gerais. Belo Horizonte: FJP.

GRANDA, E. \& BREILH, J., 1989. Saúde na Sociedade. Rio de Janeiro: Editora Cortez/ABRASCO.

IBGE (Fundação Instituto Brasileiro de Geografia e Estatística), 1996. Contagem Populacional/1996, CD-ROM. Belo Horizonte: IBGE.

MALTA, D. C.; FERREIRA, L. M.; REIS, A. T. \& MERHY, E. E., 1998. Acolhimento - Mudando o processo de trabalho em saúde, um relato da experiência em Belo Horizonte. In: O Sistema Único de Saúde em Belo Horizonte, Reescrevendo o Público (C. R. Campos, D. C. Malta, A. T. Reis, A. F. Santos \& E. E. Merhy, org.), pp. 121-142, Belo Horizonte: Editora Xamã VM.

MAPINFO CORPORATION, 1994. Map Info. New York: MapInfo Corporation

MENDES, E. V., 1994. As políticas de saúde no Brasil nos anos 80: A conformação da reforma sanitária e a construção da hegemonia do projeto neoliberal. In: Distrito Sanitário, o Processo Social de Mudança das Práticas Sanitárias do Sistema Único de Saúde (E.V. Mendes, org.), pp. 19-91, São Paulo: Editora Hucitec.

MONTEIRO, C. A., 1988. Saúde e Nutrição das Crianças de São Paulo: Diagnóstico, Contrastes Sociais e Tendências. São Paulo: Editora Hucitec/Edusp.

MONTEIRO, C. A.; BENÍCIO, M. H. D. A. \& BALDIJÃO, M. F. A., 1980. Mortalidade no primeiro anos de vida e a distribuição de renda e de recursos públicos de saúde, São Paulo, Brasil. Revista de Saúde Pública, 14:515-529.

MONTEIRO, C. A. \& NAZÁRIO, C. L., 1995. Declínio da mortalidade infantil e equidade social: O caso de São Paulo entre 1973 e 1993. In: Velhos e Novos Males da Saúde no Brasil. A Evolução do País e de suas Doenças (C. A Monteiro, org.), pp. 173-185,
São Paulo: Editora Hucitec/Núcleo de Pesquisas Epidemiológicas em Nutrição e Saúde, Universidade de São Paulo.

NAJAR, A. L. \& MARQUES, E. C., 1998. Saúde e Espaço, Estudos Metodológicos e Técnicos de Análise. Rio de Janeiro: Editora Fiocruz.

PAIM, J. S.; COSTA, M. C. N.; CABRAL, V.; MOTA, I. A. \& NEVES, R. B. B., 1987. Spatial distribution of proportional infant and certain socioeconomic variables in Salvador, Bahia, Brazil. Bulletin PAHO, 27:1-14.

RODRIGUES, S. C.; MAGALHÃES, H. M. J.; EVANGELISTA, P. A.; LADEIRA, R. M. \& LAUDARES, S., 1997. Perfil dos nascidos vivos no Município de Belo Horizonte, 1992-1994. Cadernos de Saúde Pública, 13:53-57.

ROSEN, G., 1994. Uma História da Saúde Pública. 2a Ed. São Paulo: Editora Unesp/Editora Hucitec/ Rio de Janeiro: ABRASCO.

SANTOS, M., 1985. Espaço e Método. São Paulo: Nobel.

SANTOS, M., 1991. Metamorfoses do Espaço Habitado. Fundamentos Teóricos e Metodológicos da Geografia. 2a Ed. São Paulo: Editora Hucitec.

SIMÕES, C. C. S. \& MONTEIRO, C. A., 1995. Tendência secular da mortalidade infantil no Brasil. In: Velhos e Novos Males da Saúde no Brasil. A Evolução do País e de suas Doenças (C. A. Monteiro, org.), pp. 153-156, São Paulo: Editora Hucitec/Núcleo de Pesquisas Epidemiológicas em Nutrição e Saúde, Universidade de São Paulo.

SIMÕES, C. C. S., 1992. O estudo dos diferenciais de mortalidade infantil segundo algumas características sócio-econômicas. In: Perfil de Crianças e Mães no Brasil. Aspectos de Saúde e Nutrição de Crianças no Brasil (Fundação Instituto Brasileiro de Geografia e Estatística \& Fundo das Nações Unidas para a Infância, org.), pp. 61-78, Rio de Janeiro: IBGE.

SMSA-BH (Secretaria Municipal de Saúde de Belo Horizonte), 1994. Projeto de Vigilância à Mortalidade Infantil. Belo Horizonte: SMSA-BH. (mimeo.)

SMSA-BH (Secretaria Municipal de Saúde de Belo Horizonte), 1997. Diagnóstico da Saúde da Criança no Município de Belo Horizonte. Belo Horizonte: Núcleo de Epidemiologia, SMSA-BH. (mimeo.)

SMPL-BH (Secretaria Municipal de Planejamento de Belo Horizonte), 1996. Índice de Qualidade de Vida Urbana, Belo Horizonte, Minas Gerais. Belo Horizonte: SMPL-BH.

SULBRANDT, J., 1994. Avaliação dos programas sociais: Uma perspectiva crítica dos modelos usuais. In: Pobreza: Uma Questão Inadiável (B. Kliksberg, org.), pp. 365-408, Brasília: Escola Nacional de Administração Pública.

UNGLERT, C. V. S., 1994. Territorialização em sistemas de saúde. In: Distrito Sanitário, o Processo Social de Mudança das Práticas Sanitárias do Sistema Único de Saúde (E. V. Mendes, org.), pp. 221235, São Paulo: Editora Hucitec.

VELOSO, B. G., 1995. Distrito Sanitário e Território Construindo a Descentralização no Município de Belo Horizonte/MG. Dissertação de Mestrado, Rio de Janeiro: Escola Nacional de Saúde Pública, Fundação Oswaldo Cruz.

VICTORA, C. G.; BARROS, F. C. \& VAUGHAN, J. P., 1988. Epidemiologia da Desigualdade. São Paulo: Editora Hucitec. 